\title{
An Approach to $\epsilon$-duality Theorems for Nonconvex Semi-infinite Multiobjective Optimization Problems
}

\author{
Do Sang Kim* and Ta Quang Son
}

\begin{abstract}
Using a scheme for solving multiobjective optimization problems via a system of corresponding scalar problems, approximate optimality conditions for a nonconvex semi-infinite multiobjective optimization problem are established. As a new approach, the scheme is developed to study the approximate duality theorems of the problem via a pair of primal-dual scalar problems. Several $\epsilon$-duality theorems are given. Furthermore, the existence theorem for almost quasi weakly $\epsilon$-Pareto solutions of the primal problem, and the existence theorem for quasi weakly $\epsilon$-Pareto solutions of the dual problem are established without any constraint qualification.
\end{abstract}

\section{Introduction}

Scalarizing methods are important tools to find optimal solutions in multiobjective optimization programming. The relevance of using scalarizing methods to solve multiobjective optimization problems is that, scalar problems can have more effective means of finding optima than vector problems can. The reader is referred to the papers [27,34], where surveys of methods for multiobjective optimization are reviewed and to the books $2,12,13,25$, , the papers [4, 24, 33] for the deeper insight into this topic. In this paper, we are interested in a scalarizing method for solving multiobjective optimization problems introduced in the Chankong-Haimes's book [5]. This scheme was presented since 1983 and it has attracted much attention by several authors for years. The reader is referred to the papers such as $3,4,26,27,35$ for details. The criteria of this scheme is to solve a vector optimization problem via a system of corresponding scalar problems. Hence, it could approach to the dual problem of a given multiobjective optimization problem via pairs of primal-dual scalar problems.

In this paper, we deal with approximate Pareto solutions for a multiobjective optimization nonconvex problem based on the method, and study its dual problem via the scheme. Related to approximate solutions in multiobjective optimization problems, there are many Received November 29, 2017; Accepted June 3, 2018.

Communicated by Jein-Shan Chen.

2010 Mathematics Subject Classification. 90C26, 49N15, 90C46.

Key words and phrases. generalized KKT condition up to $\epsilon$, almost quasi $\epsilon$-Pareto solution, almost quasi weakly $\epsilon$-Pareto solution, Wolfe duality.

*Corresponding author. 
papers published for years $1,8,14,15,18,21,32$. In our results, we are interested in the concepts of quasi $\epsilon$-Pareto solutions and almost quasi (weakly) $\epsilon$-Pareto solutions. We note that the concepts of a quasi $\epsilon$-solution and an almost quasi $\epsilon$-solution for a single objective nonconvex problem were considered in 31. Afterward, it was developed for multiobjective problems 32 . There are several papers concerned with these concepts $[11,22,30,38,41]$.

Let us consider the following nonconvex semi-infinite multiobjective optimization problem:

(MP) Minimize $f(x):=\left(f_{1}(x), f_{2}(x), \ldots, f_{m}(x)\right)$ subject to $g_{t}(x) \leq 0, t \in T, x \in C$,

where $f_{i}: \mathbb{R}^{n} \rightarrow \mathbb{R}$ and $g_{t}$ are locally Lipschitz functions for $i \in M:=\{1,2, \ldots, m\}$ and $t \in T, T$ is an index set (possibly infinite), and $C$ is a closed convex subset of $\mathbb{R}^{n}$. The optimization problems which have a number of infinite constraints are attracted, recently, by several authors $6,7,36,41]$. We also note that the results on approximate Pareto solutions and approximate duality theorems for $(\mathrm{MP})$ via a non-weighted sum scalarizing method were established recently in 38. Due to the scheme introduced by ChankongHaimes, for $j \in M$ and given $y \in C$, we associate to $(\mathrm{MP})$ the following scalar problem.

$\left(P_{j}(y)\right)$

Minimize $f_{j}(x)$ subject to $f_{k}(x) \leq f_{k}(y)$,

$$
k \in M^{j}:=M \backslash\{j\}, g_{t}(x) \leq 0, t \in T, x \in C,
$$

where $f_{j}, f_{k}, g_{t}$ and $C$ are as above with $k \in M^{j}$ and $t \in T$. In this research, the scheme will be developed to establish relationships between approximate optimality solutions of (MP) and $\left(P_{j}(y)\right.$.

As far as we know, the scheme is usually used for finding exact solutions of multiobjective optimization problems and there is no work dealing with dual problems of vector optimization problems. The aim of this research is to propose an approach for establishing the relations between a vector optimization problem and its dual problem via a pair of primal-dual scalar problems corresponding Chankong-Haimes scheme. So, the relations between $(\mathrm{MP})$ and its dual problem will be investigated via the following diagram

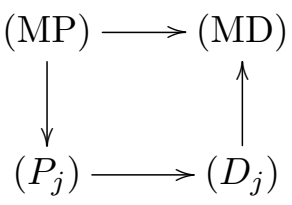

where $(\mathrm{MD})$ is the dual problem of $\left(\mathrm{MP}, D_{j}\right)$ is the dual problem of $\left(P_{j}\right),\left(P_{j}\right)$ and $\left(D_{j}\right)$ are the scalar problems corresponding to $(\mathrm{MP})$ and $(\mathrm{MD})$, respectively. When approaching the problem from this direction, there exists a question: How to formulate the problem $\left(D_{j}\right.$ ) such that it is a dual problem of $\left(P_{j}\right)$ and it can be suitable for $\mathrm{MD}$ in ChankongHaimes scheme, simultaneously. This problem will be discussed in Section 4. 
Our results are divided in two parts. In the first one, we deal with approximate Pareto solutions of $(\mathrm{MP})$. Some approximate optimality conditions for $\mathrm{MP}$ are given. The obtained results also describe the relationships between approximate Pareto solutions of (MP) and approximate solutions of $\left(P_{j}(y)\right.$. In particular, we prove that there exists an almost quasi approximate Pareto solution for (MP) without assuming any constraint qualification. In the second part, we propose an approximate dual problem of (MP) in Wolfe type. To obtain new results related to the dual problem (MD), we give some theorems to describe the relationships between $\left(P_{j}(y)\right)$ and $\left(D_{j}\right)$, between $\left(D_{j}\right)$ and $(\mathrm{MD})$. In this case, the problem $\left[D_{j}\right]$ is formulated in Wolfe type with a small modification in comparison with the original one. Then, duality relationships, between (MP) and (MD), can be investigated via a pair primal-dual scalar problems. We also prove that there exists a quasi weakly approximate Pareto solution for (MD) without assuming any constraint qualification.

The rest of the paper is organized as follows. The next section is devoted to preliminaries and lemmas for describing the criteria of the method. In the third section, we present approximate optimality conditions for $(\overline{\mathrm{MP}})$ and $\sqrt{P_{j}(y)}$ and the relationships between the problems. In the last section, approximate duality theorems for $\left(D_{j}\right)$ and $(\mathrm{MD})$ and relationships between the problems are established. Finally, relations between $(\overline{\mathrm{MP}})$ and (MD are exploited via a pair of scalar problems. An example is given and applications into fractional semi-infinite multiobjective optimization problems are discussed.

\section{Preliminaries}

Most of the following basic concepts are concerned with nonsmooth analysis theory. They can be found in the following books $9,10,16$.

A function $f: \mathbb{R}^{n} \rightarrow \mathbb{R}$ is said to be a locally Lipschitz function if for any $x \in \mathbb{R}^{n}$ there exists a positive constant $K$ and a neighborhood $N$ of $x$ such that

$$
|f(y)-f(z)| \leq K\|y-z\|, \quad \forall y, z \in N(x) .
$$

For a sequence $\left(g_{t}\right)_{t \in T}, g_{t}: \mathbb{R}^{n} \rightarrow \mathbb{R}$, we say that $\left(g_{t}\right)_{t \in T}$ is locally Lipschitz with respect to $x$ uniformly in $t$ if there exists a neighborhood $N(x)$ and a constant $K>0$ such that

$$
\left|g_{t}(y)-g_{t}(z)\right| \leq K\|y-z\|, \quad \forall y, z \in N(x) \text { and } \forall t \in T .
$$

In this research, the constraint functions $g_{t}, t \in T$, of $\mathrm{MP}$ are locally Lipschitz with respect to $x$ uniformly in $t$.

The generalized directional derivative of $f$ at $x$ in the direction $d \in X$ is defined by $($ see $[9])$

$$
f^{\circ}(x ; d):=\limsup _{\substack{h \rightarrow 0 \\ t \downarrow 0}} \frac{f(x+h+t d)-f(x+h)}{t}
$$


and the Clarke's subdifferential of $f$ at $x$, denoted by $\partial^{c} f(x)$, is

$$
\partial^{c} f(x):=\left\{u \in \mathbb{R}^{n} \mid u(d) \leq f^{\circ}(x ; d), \forall d \in \mathbb{R}^{n}\right\} .
$$

For $d \in \mathbb{R}^{n}$, if the limit

$$
\lim _{t \downarrow 0} \frac{f(x+t d)-f(x)}{t}
$$

exists then it is called the directional derivative of $f$ at $x$ in the direction $d$ and is denoted by $f^{\prime}(x ; d)$. The function $f$ is said to be quasidifferentiable or regular at $x$ (in the sense of Clarke) if $f^{\prime}(x ; d)$ exists and equals to $f^{\circ}(x ; d)$ for each $d \in \mathbb{R}^{n}$ (see 9,10 ). The function $f$ is said to be regular if it is regular at each point of $\mathbb{R}^{n}$.

For a closed subset $D$ of $\mathbb{R}^{n}$, the tangent cone to $D$ is defined by

$$
T_{D}(x)=\left\{h \in \mathbb{R}^{n} \mid d_{D}^{\circ}(x ; h)=0\right\},
$$

where $d_{D}$ denotes the distance function to $D$. The normal cone to $D$ is defined by

$$
N_{D}(x)=\left\{u \in \mathbb{R}^{n} \mid u(h) \leq 0, \forall h \in T_{D}(x)\right\} .
$$

When $D$ is convex, the normal cone to $D$ coincides with the one in the sense of convex analysis:

$$
N_{D}(x)=\left\{u \in \mathbb{R}^{n} \mid u(y-x) \leq 0, \forall y \in D\right\} .
$$

The following definitions are due to Hiriart-Urruty [23].

Definition 2.1. A locally Lipschitz function $f: \mathbb{R}^{n} \rightarrow \mathbb{R}$ is said to be pseudoconvex if for all $x, y \in \mathbb{R}^{n}$,

$$
f^{\circ}(x ; y-x) \geq 0 \quad \Longrightarrow \quad f(y) \geq f(x) .
$$

Remark 2.2. From Definition 2.1, if there exists $u \in \partial^{c} f(x)$ such that $u(y-x) \geq 0$ then $f(y) \geq f(x)$.

Definition 2.3. A locally Lipschitz function $f: \mathbb{R}^{n} \rightarrow \mathbb{R}$ is said to be quasiconvex if for all $x, y \in \mathbb{R}^{n}$,

$$
f(y) \leq f(x) \quad \Longrightarrow \quad f^{\circ}(x ; y-x) \leq 0 .
$$

We note that if $f$ is pseudoconvex on $C$ then $f$ is quasiconvex on $C$. Moreover, a quasiconvex function is characterized by the convexity of its lower-level sets (see [16]).

Definition 2.4. Let $C$ be a subset of $\mathbb{R}^{n}$ and let $\epsilon>0$. A locally Lipschitz function $f: \mathbb{R}^{n} \rightarrow \mathbb{R}$ is said to be $\epsilon$-pseudoconvex at $x \in C$ if

$$
d \in \mathbb{R}^{n}, x+d \in C, f^{\circ}(x ; d)+\sqrt{\epsilon}\|d\| \geq 0 \quad \Longrightarrow \quad f(x+d)+\sqrt{\epsilon}\|d\| \geq f(x) .
$$

The function $f$ is said to be $\epsilon$-pseudoconvex on $C$ if $f$ is $\epsilon$-pseudoconvex at every $x \in C$. 
We also note that, in Definition 2.4. if $f$ is supposed further to be regular at $x$ then $f$ is said to be $\epsilon$-semiconvex at $x$ (see 31,41$]$ ).

We now recall some concepts of approximate solutions for a single objective optimization problem. Let us consider the following scalar optimization problem.

$$
\text { Minimize } f(x) \text { subject to } g_{t}(x) \leq 0, t \in T, x \in C \text {, }
$$

where $f: \mathbb{R}^{n} \rightarrow \mathbb{R}$ is locally Lipschitz function and the functions $g_{t}, t \in T$, and $C$ are as above. The feasible set of $(\mathrm{P})$ is denoted by

$$
F:=\left\{x \in C \mid g_{t}(x) \leq 0, t \in T\right\} .
$$

Let $\alpha>0$. We denote by $F^{\alpha}$ the $\alpha$-feasible set of $(\mathrm{P})$,

$$
F^{\alpha}:=\left\{x \in C \mid g_{t}(x) \leq \sqrt{\alpha}, t \in T\right\}
$$

The following notions come from 31 .

Definition 2.5. Let $\alpha>0$. A point $z \in F^{\alpha}$ is said to be

(i) an almost $\alpha$-solution of $(\mathrm{P})$ if $f(z) \leq f(x)+\alpha$ for all $x \in F$;

(ii) an almost $\alpha$-quasisolution of $(\mathrm{P})$ if $f(z) \leq f(x)+\sqrt{\alpha}\|x-z\|$ for all $x \in F$;

(iii) an almost regular $\alpha$-solution of $(\mathrm{P})$ if it is an almost $\alpha$-solution and an almost $\alpha$-quasisolution of $(\mathrm{P})$.

The word "almost" is removed if $z \in F$.

We note that the existence theorem for almost approximate solutions of nonconvex programming problems was given in 31, 38, 41.

The following linear space is proposed firstly in [28] and is used for semi-infinite programming 17]:

$$
\mathbb{R}^{(T)}:=\left\{\lambda=\left(\lambda_{t}\right)_{t \in T} \mid \lambda_{t}=0 \text { for all } t \in T \text { but only finitely many } \lambda_{t} \neq 0\right\} .
$$

The nonnegative cone of $\mathbb{R}^{(T)}$ is denoted by

$$
\mathbb{R}_{+}^{(T)}=\left\{\lambda=\left(\lambda_{t}\right)_{t \in T} \in \mathbb{R}^{(T)} \mid \lambda_{t} \geq 0, t \in T\right\}
$$

With $\lambda \in \mathbb{R}^{(T)}$, its supporting set

$$
T(\lambda)=\left\{t \in T \mid \lambda_{t} \neq 0\right\}
$$

is a finite subset of $T$. 
Whenever $T(\lambda)=\emptyset$, we assume $\sum_{t \in T} \lambda_{t} \vartheta_{t}=0$ for any sequence $\left\{\vartheta_{t}\right\}_{t \in T}$.

For $\lambda \in \mathbb{R}^{(T)}$, we define

$$
\|\lambda\|_{1}:=\sum_{t \in T(\lambda)}\left|\lambda_{t}\right| .
$$

Let $x \in \mathbb{R}^{n}$. We need the following condition

$$
\exists d \in T_{C}(x): g_{t}^{\circ}(x ; d)<0 \text { for all } t \in I(x):=\left\{t \in T \mid g_{t}(x)=0\right\}
$$

We use $B^{*}$ to denote a closed unit ball in $\mathbb{R}^{n}$. According to Theorems 4.1 and 4.2 presented in [41] (where the problem $(\mathrm{P})$ is defined on a Banach space), we derive the following theorems for the case of the involved functions are defined on $\mathbb{R}^{n}$ and the index set $T$ is compact. The proofs can be omitted.

Theorem 2.6. Let $\alpha>0$ and let $z$ be an $\alpha$-quasisolution for $(\sqrt{\mathrm{P}})$. Assume that the condition (A) holds for $z$. Then there exists $\lambda \in \mathbb{R}_{+}^{(T)}$ such that

$$
0 \in \partial^{c} f(z)+\sum_{t \in T} \lambda_{t} \partial^{c} g_{t}(z)+N_{C}(z)+\sqrt{\alpha} B^{*}, \quad g_{t}(z)=0, \quad \forall t \in T(\lambda) .
$$

Definition 2.7. A pair $(z, \lambda) \in C \times \mathbb{R}_{+}^{(T)}$ is called a generalized KKT pair up to $\alpha>0$ if the following condition holds

$$
0 \in \partial^{c} f(z)+\sum_{t \in T} \lambda_{t} \partial^{c} g_{t}(z)+N_{C}\left(x_{\alpha}\right)+\sqrt{\alpha} B^{*}, \quad g_{t}(z) \geq 0, \quad \forall t \in T(\lambda)
$$

It was called a strict pair up to $\alpha$ if $(2.1)$ is satisfied with $g_{t}(z)>0$ for all $t \in T(\lambda)$, it is equivalent to $\lambda_{t}=0$ if $g_{t}(z) \leq 0$.

Theorem 2.8. Let $\alpha>0$. There exists an almost regular $\alpha$-solution $z \in F^{\alpha}$ for $(\sqrt{\mathrm{P}})$ and $\lambda \in \mathbb{R}_{+}^{(T)}$ such that $(z, \lambda)$ is a strict generalized KKT pair up to $\alpha$.

For the problem $(\mathrm{MP})$, its feasible set is also denoted by $F$. Let $\epsilon=\left(\epsilon_{1}, \ldots, \epsilon_{m}\right) \in \mathbb{R}_{++}^{m}$ with $\mathbb{R}_{++}^{m}$ denoting the positive orthant of $\mathbb{R}^{m}$. Set

$$
\theta:=\min _{i \in M} \epsilon_{i}
$$

and

$$
F^{\theta}:=\left\{x \in C \mid g_{t}(x) \leq \sqrt{\theta}, t \in T\right\}
$$

The following concepts are due to the ones presented in [30] (see also [38]).

Definition 2.9. A point $z \in F^{\theta}$ is said to be 
(i) an almost $\epsilon$-Pareto solution of $(\mathrm{MP})$ if there exists no $x \in F$ such that

$$
f_{i}(x) \leq f_{i}(z)-\epsilon_{i}, \quad i \in M
$$

with at least one strict inequality;

(ii) an almost $\epsilon$-Pareto quasisolution of (MP) if there exists no $x \in F$ such that

$$
f_{i}(x)+\sqrt{\epsilon_{i}}\|x-z\| \leq f_{i}(z), \quad i \in M
$$

with at least one strict inequality;

(iii) an almost regular $\epsilon$-Pareto solution of (MP) if it is an almost $\epsilon$-Pareto solution of (MP) and is an almost $\epsilon$-Pareto quasisolution of (MP).

If $z \in F$ then we have the concepts of $\epsilon$-Pareto solution, $\epsilon$-Pareto quasisolution, and regular $\epsilon$-Pareto solution of $(\mathrm{MP}$, respectively.

Definition 2.10. A point $z \in F^{\theta}$ is said to be

(i) an almost weakly $\epsilon$-Pareto solution of $(\mathrm{MP})$ if there exists no $x \in F$ such that

$$
f_{i}(x)<f_{i}(z)-\epsilon_{i}, \quad i \in M
$$

(ii) an almost weakly $\epsilon$-Pareto quasisolution of $(\mathrm{MP})$ if there exists no $x \in F$ such that

$$
f_{i}(x)+\sqrt{\epsilon_{i}}\|x-z\|<f_{i}(z), \quad i \in M
$$

(iii) an almost regular weakly $\epsilon$-Pareto solution of $(\mathrm{MP})$ if it is an almost weakly $\epsilon$-Pareto solution of $(\mathrm{MP})$ and is an almost weakly $\epsilon$-Pareto quasisolution of $(\mathrm{MP})$.

If $z \in F$ then we have the concepts of weakly $\epsilon$-Pareto solution, weakly $\epsilon$-Pareto quasisolution, and regular weakly $\epsilon$-Pareto solution of (MP), respectively.

The criteria of Chankong-Haimes scheme (see also [4, 27]) applied for (MP) is that: A feasible point $z$ of $(\mathrm{MP})$ is a Pareto solution of $(\mathrm{MP})$ if and only if it is an optimal solution of $\left(P_{j}(z)\right)$ for each $j \in M$.

For a problem $P_{j}(y), j \in M$, its feasible set is denoted by $F_{j}(y)$. Set $M^{j}:=M \backslash\{j\}$. The method above is developed for finding approximate Pareto solutions of (MP) as follows. Given $y \in C, j \in M$ and $\epsilon_{j}>0$, the $\epsilon_{j}$-feasible set of $\left[P_{j}(y)\right.$ is defined by

$$
F_{j}^{\epsilon_{j}}(y):=\left\{x \in C \mid f_{k}(x) \leq f_{k}(y)+\sqrt{\epsilon_{j}}, k \in M^{j} ; g_{t}(x) \leq \sqrt{\epsilon_{j}}, t \in T\right\} .
$$

From the criteria of Chankong-Haimes scheme discussed above, it is easy to derive the following lemmas. 
Lemma 2.11. Let $\epsilon \in \mathbb{R}_{++}^{m}$.

(i) A point $z$ is an almost $\epsilon$-Pareto solution of (MP if and only if $z$ is an almost $\epsilon_{j}$-solution of $\left(P_{j}(z)\right)$ for each $j \in M$.

(ii) A point $z$ is an almost $\epsilon$-Pareto quasisolution of $(\mathrm{MP}$ if and only if $z$ is an almost $\epsilon_{j}$-quasisolution of $\left(P_{j}(z)\right)$ for each $j \in M$.

(iii) A point $z$ is an almost regular $\epsilon$-Pareto solution of (MP) if and only if $z$ is an almost regular $\epsilon_{j}$-solution of $\left(P_{j}(z)\right)$ for each $j \in M$.

Lemma 2.12. Let $\epsilon \in \mathbb{R}_{++}^{m}$ and let $j \in M$ be such that $\epsilon_{j}=\min _{i \in M} \epsilon_{i}$.

(i) A point $z$ is an almost weakly $\epsilon$-Pareto solution of $(\mathrm{MP}$ if and only if $z$ is an almost $\epsilon_{j}$-solution of $\left(P_{j}(z)\right)$.

(ii) A point $z$ is an almost weakly $\epsilon$-Pareto quasisolution of $\mathrm{MP}$ if and only if $z$ is an almost $\epsilon_{j}$-quasisolution of $\left(P_{j}(z)\right)$.

(iii) A point $z$ is an almost regular weakly $\epsilon$-Pareto solution of (MP) if and only if $z$ is an almost regular $\epsilon_{j}$-solution of $\left(P_{j}(z)\right)$.

Remark 2.13. In Lemmas 2.11 and 2.12, the conclusions are valid if we remove the word "almost".

Example 2.14. The following example illustrates relations between approximate solutions of $(\mathrm{MP})$ and approximate solutions of scalar problems corresponding to ChankongHaimes method

$(\mathrm{MP} 1) \quad \operatorname{Min} f(x)=\left(x, x^{2}-x\right)$ subject to $g(x)=t x^{2}-x \leq 0, t \in[0,1], x \in[-1,1]$.

Let $f_{1}(x)=x$ and $f_{2}(x)=x^{2}-x$. Observe that the feasible set of (MP1) is $[0,1]$. Given $\epsilon=(1 / 2,1 / 2)$, it is clear that $z=1 / 2$ is $\epsilon$-Pareto solution of (MP1).

The two scalar problems corresponding to (MP1) in Chankong-Haimes method are formulated as follows:

$\left(P_{1}(z)\right)$

Min $f_{1}(x)=x$ subject to $x^{2}-x \leq f_{2}(z)=-1 / 4, t x^{2}-x \leq 0, t \in[0,1], x \in[-1,1]$

and

$\left(P_{2}(z)\right)$

Min $f_{2}(x)=x^{2}-x$ subject to $x \leq f_{1}(z)=1 / 2, t x^{2}-x \leq 0, t \in[0,1], x \in[-1,1]$.

For the problem $\left(P_{1}(z)\right.$, with a simple computation, its feasible set is $F_{1}=\{1 / 2\}$. On the other hand, we also have inf $\left[P_{1}(z)=0\right.$. Moreover, we can see $f(z) \leq \inf \left(P_{1}(z)+\epsilon_{1}\right.$. 
Hence, $z=1 / 2$ is an $\epsilon_{1}$-solution of $\left(P_{1}(z)\right.$. For the problem $\left(P_{2}(z)\right.$, its feasible set is $F_{2}=\{x \mid 0 \leq x \leq 1 / 2\}$ and its optimal value is $\inf \left(P_{2}(z)=-1 / 4\right.$. We can see that $f(z) \leq \inf \left(P_{2}(z)+\epsilon_{2}\right.$. Thus, $z=1 / 2$ is also an $\epsilon_{2}$-solution of $P_{2}(z)$.

\section{3. $\epsilon$-optimality conditions}

We now establish $\epsilon$-optimality conditions for $\mathrm{MP}$. The following condition is associated to the problem $\left(P_{j}(z)\right)$.

Let $x \in \mathbb{R}^{n}, I(x)=\left\{t \in T \mid g_{t}(x)=0\right\}, H_{j}(x)=\left\{k \in M^{j} \mid f_{k}(x)=f_{k}(z)\right\}$, $M^{j}:=M \backslash\{j\}$ and $\bar{T}(x)=I(x) \cup H_{j}(x)$.

$$
\exists d \in T_{C}(x): \begin{cases}g_{t}^{\circ}(x ; d)<0 & \text { for all } t \in I(x), \\ f_{k}^{\circ}(x ; d)<0 & \text { for all } k \in H_{j}(x) .\end{cases}
$$

By applying Theorem 2.6 for the problem $\left(P_{j}(z)\right)$, we can obtain the following result. The proof is omitted.

Proposition 3.1. Let $\epsilon_{j}>0$ and let $z$ be an $\epsilon_{j}$-quasisolution for $\left(P_{j}(z)\right)$. Assume that the condition $\left(\mathcal{A}_{j}\right.$ holds for $z$. Then there exist $\mu_{k} \geq 0, k \in M^{j}$ and $\lambda \in \mathbb{R}_{+}^{(T)}$ such that

$$
\begin{gathered}
0 \in \partial^{c} f_{j}(z)+\sum_{k \in M^{j}} \mu_{k} \partial^{c} f_{k}(z)+\sum_{t \in T} \lambda_{t} \partial^{c} g_{t}(z)+N_{C}(z)+\sqrt{\epsilon_{j}} B^{*}, \\
g_{t}(z)=0, \quad \forall t \in T(\lambda) .
\end{gathered}
$$

We need the following proposition. The proof is omitted.

Proposition 3.2. Let $\epsilon_{j}>0$ and let $z \in F_{j}(z)$. Assume that the function $f_{j}$ is $\epsilon_{j}$ pseudoconvex, the functions $f_{k}$ and $g_{t}$ are quasiconvex where $k \in M^{j}$ and $t \in T$. If there exist $\mu_{k} \geq 0, k \in M^{j}$ and $\lambda \in \mathbb{R}_{+}^{(T)}$ such that

$$
\begin{gathered}
0 \in \partial^{c} f_{j}(z)+\sum_{k \in M^{j}} \mu_{k} \partial^{c} f_{k}(z)+\sum_{t \in T} \lambda_{t} \partial^{c} g_{t}(z)+N_{C}(z)+\sqrt{\epsilon_{j}} B^{*} \\
g_{t}(z)=0, \quad \forall t \in T(\lambda)
\end{gathered}
$$

then $z$ is an $\epsilon_{j}$-quasisolution for $\left(P_{j}(z)\right)$.

In the following theorem, a necessary condition for an $\epsilon$-Pareto quasisolution of $(\mathrm{MP}$ can be obtained if there exists $j \in M$ such that the condition $\left(\mathcal{A}_{j}\right.$ holds.

Theorem 3.3. Let $\in \in \mathbb{R}_{++}^{m}$. Let $z \in F$ be an $\epsilon$-Pareto quasisolution of (MP). If there exists $j \in M$ such that the condition $\left(\mathcal{A}_{j}\right.$ ) holds for $z$ then there exist $\bar{\mu}_{k} \geq 0, k \in M$, $\sum_{k \in M} \bar{\mu}_{k}=1$ and $\bar{\lambda} \in \mathbb{R}_{+}^{(T)}$ such that

$$
0 \in \sum_{k \in M} \bar{\mu}_{k} \partial^{c} f_{k}(z)+\sum_{t \in T} \bar{\lambda}_{t} \partial^{c} g_{t}(z)+N_{C}(z)+\sqrt{\epsilon_{j}} B^{*}, \quad g_{t}(z)=0, \forall t \in T(\bar{\lambda}) .
$$


Proof. Note that if $z$ is an $\epsilon$-Pareto quasisolution of (MP then, by Lemma 2.11 and Remark 2.13, $z$ is an $\epsilon_{j}$-quasisolution $\left(P_{j}(z)\right)$. Since the condition $\left(\mathcal{A}_{j}\right)$ holds for $z$, by Proposition 3.1, there exist $\mu_{k} \geq 0, k \in M^{j}$ and $\lambda \in \mathbb{R}_{+}^{(T)}$ such that

$$
\begin{gathered}
0 \in \partial^{c} f_{j}(z)+\sum_{k \in M^{j}} \mu_{k} \partial^{c} f_{k}(z)+\sum_{t \in T} \lambda_{t} \partial^{c} g_{t}(z)+N_{C}(z)+\sqrt{\epsilon_{j}} B^{*}, \\
g_{t}(z)=0, \quad \forall t \in T(\lambda),
\end{gathered}
$$

where $B^{*}$ is an unit ball in $\mathbb{R}^{n}$. This implies that

$$
0 \in \frac{1}{1+\sum_{k \in M^{j}} \mu_{k}}\left(\partial^{c} f_{j}(z)+\sum_{k \in M^{j}} \mu_{k} \partial^{c} f_{k}(z)+\sum_{t \in T} \lambda_{t} \partial^{c} g_{t}(z)+N_{C}(z)+\sqrt{\epsilon_{j}} B^{*}\right),
$$

and $g_{t}(z)=0, \forall t \in T(\lambda)$. Set

$$
\bar{\mu}_{j}=\frac{1}{1+\sum_{k \in M^{j}} \mu_{k}}, \quad \bar{\mu}_{k}=\frac{\mu_{k}}{1+\sum_{k \in M^{j}} \mu_{k}}, \quad \bar{\lambda}_{t}=\frac{\lambda_{t}}{1+\sum_{k \in M^{j}} \mu_{k}} .
$$

Note that

$$
\frac{1}{1+\sum_{k \in M^{j}} \mu_{k}} N_{C}(z) \subset N_{C}(z) \text { and } \frac{1}{1+\sum_{k \in M^{j}} \mu_{k}} \sqrt{\epsilon_{j}} B^{*} \subset \sqrt{\epsilon_{j}} B^{*} .
$$

From 3.2 , we deduce that

$$
0 \in \sum_{k \in M} \bar{\mu}_{k} \partial^{c} f_{k}(z)+\sum_{t \in T} \bar{\lambda}_{t} \partial^{c} g_{t}(z)+N_{C}(z)+\sqrt{\epsilon_{j}} B^{*}, \quad g_{t}(z)=0, \forall t \in T(\bar{\lambda}) .
$$

It is easy to check that $\sum_{k \in M} \bar{\mu}_{k}=1$.

The reader is referred to [41], where a strict KKT condition is proposed corresponding to an almost approximate solution of a single objective nonconvex optimization problem with an infinite number of constraints, and is referred to [11,29 where various type of constraint qualifications are required to study KKT conditions for multiobjective optimization problems with inequality constraints.

We now prove that there exists an almost weakly $\epsilon$-Pareto quasisolution for the problem (MP without assuming any constraint qualification. To start with, firstly, we give the definition of a so-called generalized $j$-KKT pair for $(\mathrm{MP}$. It is modified from the one in 41. Then we prove that there exists an almost regular $\epsilon_{j}$-solution for $\left(P_{j}(z)\right)$.

Definition 3.4. Let $\epsilon \in \mathbb{R}_{++}^{m}$. A pair $(z, \lambda) \in C \times \mathbb{R}_{+}^{(T)}$ is said to be a generalized $j$-KKT pair up to $\epsilon_{j}$ if the following condition is satisfied

$$
0 \in \partial^{c} f_{j}(z)+\sum_{t \in T} \lambda_{t} \partial^{c} g_{t}(z)+N_{C}(z)+\sqrt{\epsilon_{j}} B^{*}, \quad g_{t}(z) \geq 0, \forall t \in T(\lambda) .
$$

It is called strict if 3.3 holds with $g_{t}(z)>0$ for all $t \in T(\lambda)$, equivalent to $\lambda_{t}=0$ if $g_{t}(z) \leq 0$. 
Lemma 3.5. Let $\epsilon \in \mathbb{R}_{++}^{m}$. For each $j \in M$, there exists an almost regular $\epsilon_{j}$-solution $z$ for $\left(P_{j}(z)\right)$ and $\bar{\lambda} \in \mathbb{R}_{+}^{(T)}$ such that the pair $(z, \bar{\lambda})$ satisfies the condition (3.3) with $g_{t}(z)>0$ for all $t \in T(\bar{\lambda})$.

Proof. Let us consider the function $G_{t}$ defined by

$$
G_{t}= \begin{cases}f_{t}-f_{t}(z) & \text { if } t \in M^{j} \\ g_{t} & \text { if } t \in T\end{cases}
$$

and $\bar{T}=T \cup M^{j}$. For $\epsilon_{j}>0$, applying Theorem 2.8 to the problem

$$
\min \left\{f_{j}(x) \mid x \in C, G_{t}(x) \leq 0, t \in \bar{T}\right\}
$$

There exists an almost regular $\epsilon_{j}$-solution $z \in F_{j}^{\epsilon_{j}}(z)$ and $\bar{\lambda} \in \mathbb{R}_{+}^{(\bar{T})}$ such that $(z, \bar{\lambda})$ satisfies strictly the generalized KKT pair up to $\epsilon_{j}$, i.e.,

$$
\begin{gathered}
0 \in \partial^{c} f_{j}(z)+\sum_{t \in M^{j}} \bar{\lambda}_{t} \partial^{c} G_{t}(z)+\sum_{t \in T} \bar{\lambda}_{t} \partial^{c} G_{t}(z)+N_{C}(z)+\sqrt{\epsilon_{j}} B^{*}, \\
G_{t}(z)>0, \quad t \in T(\bar{\lambda}) .
\end{gathered}
$$

Since $G_{t}(z)=0$ for all $t \in M^{j}$ and $(z, \bar{\lambda})$ satisfies strictly a generalized KKT pair up to $\epsilon_{j}$, by Definition 2.7, we get $\bar{\lambda}_{t}=0$ for all $t \in M^{j}$. This and (3.4) imply

$$
0 \in \partial^{c} f_{j}(z)+\sum_{t \in T} \bar{\lambda}_{t} \partial^{c} g_{t}(z)+N_{C}(z)+\sqrt{\epsilon_{j}} B^{*}, \quad g_{t}(z)>0, \forall t \in T(\bar{\lambda}) .
$$

The desired result is proved.

Corollary 3.6. Let $\epsilon \in \mathbb{R}_{++}^{m}$ and let $j \in M$ be such that $\epsilon_{j}=\min \left\{\epsilon_{i}, i \in M\right\}$. Then there exist an almost regular weakly $\epsilon$-Pareto solution $z$ for $\mathrm{MP}$ and $\bar{\lambda} \in \mathbb{R}_{+}^{(T)}$ such that $(z, \bar{\lambda})$ satisfies strictly a generalized $j$-KKT pair up to $\epsilon_{j}$.

Proof. Indeed, by combining Lemmas 2.12 and 3.5, one can guarantee that there exists an almost regular weakly $\epsilon$-Pareto solution $z$ for $(\mathrm{MP}$.

From the corollary above, we propose a sufficient condition for an almost weakly $\epsilon$ Pareto quasisolution for $(\mathrm{MP})$ as follows.

Theorem 3.7. Let $\epsilon \in \mathbb{R}_{++}^{m}$ and let $j \in M$ be such that $\epsilon_{j}=\min \left\{\epsilon_{k}, k \in M\right\}$. Suppose that the function $f_{j}$ is $\epsilon_{j}$-pseudoconvex, the functions $g_{t}$ are quasiconvex for all $t \in T$, and the pair $(z, \lambda) \in C \times \mathbb{R}_{+}^{(T)}$ satisfies the generalized $j-K K T$ condition up to $\epsilon_{j}$ given by (3.3).

(i) If $z \in F_{j}^{\epsilon_{j}}(z)$, then $z$ is an almost weakly $\epsilon$-Pareto quasisolution of $\mathrm{MP}$.

(ii) If $z \in F_{j}(z)$, then $z$ is a weakly $\epsilon$-Pareto quasisolution of $\mathrm{MP}$. 
Proof. (i) Let $(z, \lambda) \in C \times \mathbb{R}_{+}^{(T)}$ be such that the condition 3.3 is satisfied. Then there exist $u_{j} \in \partial^{c} f_{j}(z), v_{t} \in \partial^{c} g_{t}(z), t \in T, w \in N_{C}(z)$ and $b \in B^{*}$ such that

$$
u_{j}+\sum_{t \in T} \lambda_{t} v_{t}+w+\sqrt{\epsilon_{j}} b=0 .
$$

Hence,

$$
u_{j}(x-z)+\sum_{t \in T} \lambda_{t} v_{t}(x-z)+\sqrt{\epsilon_{j}}\|x-z\|=-w(x-z) \geq 0, \quad \forall x \in C .
$$

On the other hand, since $g_{t}(z) \geq 0$ for all $t \in T(\lambda)$, and $g_{t}$ are quasiconvex functions, we can deduce that $v_{t}(x-z) \leq 0$ for all $t \in T$ and for all $x \in F$. Consequently,

$$
u_{j}(x-z)+\sqrt{\epsilon_{j}}\|x-z\| \geq 0, \quad \forall x \in F .
$$

If $z \in F_{j}^{\epsilon_{j}}(z)$ then $z \in F^{\theta}$. We claim that $z$ is an almost weakly $\epsilon$-Pareto quasisolution for $\mathrm{MP}$. On the contrary, if $z$ is not an almost weakly $\epsilon$-Pareto quasisolution of $(\mathrm{MP})$, then there exists $x \in F$ such that

$$
f_{i}(x)+\sqrt{\epsilon_{i}}\|x-z\|<f_{i}(z), \quad \forall i \in M .
$$

This implies that

$$
f_{j}(x)+\sqrt{\epsilon_{j}}\|x-z\|<f_{j}(z) .
$$

Since $f_{j}$ is $\epsilon_{j}$-pseudoconvex, the inequality above implies

$$
f_{j}^{\circ}(z ; x-z)+\sqrt{\epsilon_{j}}\|x-z\|<0 .
$$

Hence,

$$
u_{j}(x-z)+\sqrt{\epsilon_{j}}\|x-z\|<0, \quad \forall u_{j} \in \partial^{c} f_{j}(z),
$$

a contradiction to 3.6 .

(ii) When $z \in F_{j}(z)$, the conclusion can be derived similarly.

\section{4. $\epsilon$-duality theorems}

Let us define the vector Lagrange function associated to $\mathrm{MP}$ by

$$
\mathcal{L}(y, \lambda)=\left(L_{1}(y, \lambda), \ldots, L_{m}(y, \lambda)\right)
$$

where $L_{j}(y, \lambda)=f_{j}(y)+\sum_{t \in T} \lambda_{t} g_{t}(y)$. For $\epsilon \in \mathbb{R}_{++}^{m}$, the approximate dual problem of (MP in Wolfe type is formulated as follows:

$$
\begin{gathered}
\operatorname{Max} \mathcal{L}(y, \lambda), \quad 0 \in \sum_{j \in M} \tau_{j} \partial^{c} f_{j}(y)+\sum_{t \in T} \lambda_{t} \partial^{c} g_{t}(y)+N_{C}(y)+\sum_{j \in M} \tau_{j} \sqrt{\epsilon_{j}} B^{*}, \\
\tau_{j} \geq 0, \quad \sum_{j \in M} \tau_{j}=1, \quad y \in C, \quad \lambda \in \mathbb{R}_{+}^{(T)} .
\end{gathered}
$$


Definition 4.1. A feasible point $(\bar{z}, \bar{\lambda}, \bar{\tau})$ of $(\mathrm{MD})$ is said to be

(i) an $\epsilon$-Pareto solution of (MD) if there exists no feasible point $(y, \lambda, \tau)$ of (MD) such that

$$
L_{i}(\bar{z}, \bar{\lambda}) \leq L_{i}(y, \lambda)-\epsilon_{i}, \quad i \in M
$$

with at least one strict inequality;

(ii) an $\epsilon$-Pareto quasisolution of (MD) if there exists no feasible point $(y, \lambda, \tau)$ of (MD) such that

$$
L_{i}(y, \lambda)-\sqrt{\epsilon_{i}}\|y-\bar{z}\|-\sqrt{\epsilon_{i}}\|\bar{\lambda}-\lambda\|_{1} \geq L_{i}(\bar{z}, \bar{\lambda}), \quad i \in M
$$

with at least one strict inequality;

(iii) a regular $\epsilon$-Pareto solution of $(\mathrm{MD})$ if it is an $\epsilon$-Pareto solution of (MD) and is an $\epsilon$-Pareto quasisolution of $(\mathrm{MD})$.

In the spirit of Definition 2.10, the corresponding notions of weakly $\epsilon$-Pareto solutions of (MD) are obtained.

To investigate duality relationships between $(\mathrm{MP})$ and $(\mathrm{MD})$, following the diagram introduced in the first section, we need a pair of primal-dual scalar problems $\left(P_{j}\right)$ and $\left(D_{j}\right)$ such that $\left(P_{j}\right)$ is associated to $(\mathrm{MP})$ and $\left(D_{j}\right)$ is associated to (MD). Let us take a glance at Lemma 3.5 . If we focus on almost $\epsilon_{j}$-quasisolutions of $\left(P_{j}\right)$ then the obtained generalized $\epsilon_{j}$-KKT conditions do not contain the constraints $f_{k}$ for $k \in M^{j}$ (compare (3.1) with (3.5)). Motivated by this observation, we can propose a formulation for an approximate dual problem $\left(D_{j}\right)$ in Wolfe type with a small modification. The small modification respect to $\left[D_{j}\right]$ is that, it has no relation to the constraint functions $f_{k}$, $k \in M^{j}$, of $\left(P_{j}\right)$. In this case, $\left(D_{j}\right)$ will be proved to be compatible with (MD). It is formulated as follows:

$$
\begin{gathered}
\operatorname{Max} L_{j}(y, \lambda)=f_{j}(y)+\sum_{t \in T} \lambda_{t} g_{t}(y) \\
0 \in \partial^{c} f_{j}(y)+\sum_{t \in T} \lambda_{t} \partial^{c} g_{t}(y)+N_{C}(y)+\sqrt{\epsilon_{j}} B^{*}, \quad(y, \lambda) \in C \times \mathbb{R}_{+}^{(T)} .
\end{gathered}
$$

\subsection{Duality relationships between $\left(P_{j}(y)\right.$ and $D_{j}$}

Let us denote by $F_{j}^{D}$ the feasible set of $D_{j}$.

Definition 4.2. Let $(\bar{z}, \bar{\lambda})$ be a feasible solution of $\left(D_{j}\right)$. The point $(\bar{z}, \bar{\lambda})$ is called

(i) an $\epsilon_{j}$-solution for $\left(D_{j}\right)$ if

$$
L_{j}(\bar{z}, \bar{\lambda}) \geq L_{j}(y, \lambda)-\epsilon_{j}, \quad \forall(y, \lambda) \in F_{j}^{D} ;
$$


(ii) an $\epsilon_{j}$-quasisolution for $\left(D_{j}\right)$ if

$$
L_{j}(\bar{z}, \bar{\lambda}) \geq L_{j}(y, \lambda)-\sqrt{\epsilon_{j}}\|\bar{z}-y\|-\sqrt{\epsilon_{j}}\|\bar{\lambda}-\lambda\|_{1}, \quad \forall(y, \lambda) \in F_{j}^{D}
$$

(iii) a regular $\epsilon_{j}$-solution for $\sqrt{D_{j}}$ if it is both an $\epsilon_{j}$-solution and an $\epsilon_{j}$-quasisolution.

The following theorem describes the approximate weak duality between $\left[P_{j}(y)\right]$ and $D_{j}$.

Theorem 4.3. Let $x$ and $(y, \lambda)$ be the feasible points of $\left(P_{j}(y)\right.$ and $\left(D_{j}\right)$, respectively. Suppose that $L_{j}(\cdot, \lambda)$ is $\epsilon_{j}$-pseudoconvex and $f_{j}, g_{t}, t \in T$, are regular on $C$. Then,

$$
f_{j}(x) \geq L_{j}(y, \lambda)-\sqrt{\epsilon_{j}}\|x-y\| .
$$

Proof. Since $(y, \lambda)$ is a feasible solution of $\left(D_{j}\right)$, there exist $u \in \partial^{c} f_{j}(y), v_{t} \in \partial^{c} g_{t}(y)$, $w \in N_{C}(y)$ and $b \in B^{*}$ such that

$$
0=u+\sum_{t \in T} \lambda_{t} v_{t}+w+\sqrt{\epsilon_{j}} b .
$$

Since $w(x-y) \leq 0$ for all $x \in F$, it is easy to deduce that

$$
u(x-y)+\sum_{t \in T} \lambda_{t} v_{t}(x-y)+\sqrt{\epsilon_{j}}\|x-y\| \geq 0, \quad \forall x \in C .
$$

Since $f_{j}, g_{t}, t \in T$, are regular on $C$ and $L_{j}(\cdot, \lambda)$ is $\epsilon_{j}$-pseudoconvex, from (4.1), we obtain

$$
L_{j}(x, \lambda)+\sqrt{\epsilon_{j}}\|x-y\| \geq L_{j}(y, \lambda), \quad \forall x \in C .
$$

On the other hand,

$$
L_{j}(x, \lambda)=f_{j}(x)+\sum_{t \in T} \lambda_{t} g_{t}(x) \leq f_{j}(x), \quad \forall x \in F .
$$

The desired result follows.

Theorem 4.4. For problem $\left(D_{j}\right)$, suppose that the $L_{j}(\cdot, \lambda)$ is $\epsilon_{j}$-pseudoconvex and $f_{j}$, $g_{t}, t \in T$, are regular on $C$. Then, there exists an $\epsilon_{j}$-quasisolution for $D_{j}$.

Proof. By Lemma 3.5 , there exists an almost $\epsilon_{j}$-quasisolution $\bar{z}$ for $\left(P_{j}(\bar{z})\right)$ and $\bar{\lambda} \in \mathbb{R}_{+}^{(T)}$ such that $(\bar{z}, \bar{\lambda})$ is a strict generalized $j$-KKT pair up to $\epsilon_{j}$, i.e.,

$$
0 \in \partial^{c} f_{j}(\bar{z})+\sum_{t \in T} \bar{\lambda}_{t} \partial^{c} g_{t}(\bar{z})+N_{C}(\bar{z})+\sqrt{\epsilon_{j}} B^{*}, \quad g_{t}(\bar{z})>0, \forall t \in T(\bar{\lambda}) .
$$

Hence, $(\bar{z}, \bar{\lambda})$ is a feasible point of $\left(D_{j}\right)$. It needs to prove that $(\bar{z}, \bar{\lambda})$ is an $\epsilon_{j}$-quasisolution for $\left.D_{j}\right)$, i.e., to prove that

$$
L_{j}(\bar{z}, \bar{\lambda}) \geq L_{j}(y, \lambda)-\sqrt{\epsilon_{j}}\|\bar{z}-y\|-\sqrt{\epsilon_{j}}\|\bar{\lambda}-\lambda\|_{1}, \quad \forall(y, \lambda) \in F_{j}^{D} .
$$


We claim that

$$
L_{j}(\bar{z}, \bar{\lambda}) \geq L_{j}(\bar{z}, \lambda)-\sqrt{\epsilon_{j}}\|\bar{\lambda}-\lambda\|_{1}, \quad \forall \lambda \in \mathbb{R}_{+}^{(T)},
$$

i.e.,

$$
\sum_{t \in T}\left(\bar{\lambda}_{t}-\lambda_{t}\right) g_{t}(\bar{z}) \geq-\sqrt{\epsilon_{j}} \sum_{t \in T}\left|\bar{\lambda}_{t}-\lambda_{t}\right| .
$$

Indeed, set $T=T(\bar{\lambda}) \cup(T \backslash T(\bar{\lambda}))$. If $t \in T(\bar{\lambda})$ then $g_{t}(\bar{z})>0$. Hence,

$$
\left(\bar{\lambda}_{t}-\lambda_{t}\right) g_{t}(\bar{z}) \geq-\left|\left(\bar{\lambda}_{t}-\lambda_{t}\right)\right| g_{t}(\bar{z}) \text {. }
$$

Since $\bar{z} \in F_{j}^{\epsilon_{j}}(\bar{z}), g_{t}(\bar{z}) \leq \sqrt{\epsilon_{j}}$. We get

$$
\left(\bar{\lambda}_{t}-\lambda_{t}\right) g_{t}(\bar{z}) \geq-\left|\left(\bar{\lambda}_{t}-\lambda_{t}\right)\right| g_{t}(\bar{z}) \geq-\left|\left(\bar{\lambda}_{t}-\lambda_{t}\right)\right| \sqrt{\epsilon_{j}} .
$$

If $t \in T \backslash T(\bar{\lambda})$ then, by the strict generalized $j$-KKT pair up to $\epsilon_{j} 4.2$, we have $\bar{\lambda}_{t}=0$ and $g_{t}(\bar{z}) \leq 0$. Hence,

$$
\left(\bar{\lambda}_{t}-\lambda_{t}\right) g_{t}(\bar{z})=-\lambda_{t} g_{t}(\bar{z}) \geq 0
$$

Thus,

$$
\left(\bar{\lambda}_{t}-\lambda_{t}\right) g_{t}(\bar{z}) \geq-\left|\left(\bar{\lambda}_{t}-\lambda_{t}\right)\right| \sqrt{\epsilon_{j}}
$$

From (4.4) and 4.5) we can deduce 4.3). On the other hand, for a feasible point $(y, \lambda)$ of $\left.D_{j}\right)$, we get

$$
0 \in \partial^{c} f_{j}(y)+\sum_{t \in T} \lambda_{t} \partial^{c} g_{t}(y)+N_{C}(y)+\sqrt{\epsilon_{j}} B^{*} .
$$

Using an argument similar to the one in the proof of Theorem 4.3, we get

$$
L_{j}(x, \lambda)+\sqrt{\epsilon_{j}}\|x-y\| \geq L_{j}(y, \lambda), \quad \forall x \in C .
$$

Hence,

$$
L_{j}(\bar{z}, \lambda) \geq L_{j}(y, \lambda)-\sqrt{\epsilon_{j}}\|\bar{z}-y\| .
$$

The desired conclusion follows by combining 4.6 and 4.3).

Remark 4.5. Suppose that the $L_{j}(\cdot, \lambda)$ is $\epsilon_{j}$-pseudoconvex and $f_{j}, g_{t}, t \in T$, are regular on $C$. If $\bar{z}$ is an almost $\epsilon_{j}$-quasisolution for $\left(P_{j}(\bar{z})\right)$ and $\bar{\lambda} \in \mathbb{R}_{+}^{(T)}$ such that $(\bar{z}, \bar{\lambda})$ is a generalized $j$-KKT pair up to $\epsilon_{j}$, then $(\bar{z}, \bar{\lambda})$ is an $\epsilon_{j}$-quasisolution of $\left(D_{j}\right)$.

Theorem 4.6. Let $(\bar{z}, \bar{\lambda})$ be a feasible solution of $\left(D_{j}\right)$. Suppose that $f_{j}$ is $\epsilon_{j}$-pseudoconvex, $g_{t}$ are quasiconvex for $t \in T$. Suppose further that $g_{t}(x) \leq g(z), t \in T$, for all $x \in F$ and $f_{j}, g_{t}$ are regular on $C$ for $t \in T$. 
(i) If $\bar{z} \in F_{j}(\bar{z})$, then $\bar{z}$ is an $\epsilon_{j}$-quasisolution of $\left(P_{j}(\bar{z})\right)$.

(ii) If $\bar{z} \in F_{j}^{\epsilon_{j}}(\bar{z})$, then $\bar{z}$ is an almost $\epsilon_{j}$-quasisolution of $\left(P_{j}(\bar{z})\right)$.

Proof. Let $(\bar{z}, \bar{\lambda})$ be a feasible solution of $\left(D_{j}\right)$. We get

$$
0 \in \partial^{c} f_{j}(\bar{z})+\sum_{t \in T} \bar{\lambda}_{t} \partial^{c} g_{t}(\bar{z})+N_{C}(\bar{z})+\sqrt{\epsilon_{j}} B^{*}
$$

Then there exist $u_{j} \in \partial^{c} f_{j}(\bar{z}), v_{t} \in \partial^{c} g_{t}(\bar{z}), t \in T, w \in N_{C}(\bar{z})$ and $b \in B^{*}$ such that

$$
u_{j}(x-\bar{z})+\sum_{t \in T} \bar{\lambda}_{t} v_{t}(x-\bar{z})+\sqrt{\epsilon_{j}}\|x-\bar{z}\|=-w(x-\bar{z}) \geq 0, \quad \forall x \in C .
$$

Since $g_{t}$ are quasiconvex and $g_{t}(x) \leq g_{t}(\bar{z})$ for all $t \in T$ and for all $x \in F$, we deduce that

$$
u_{j}(x-\bar{z})+\sqrt{\epsilon_{j}}\|x-\bar{z}\| \geq 0, \quad \forall x \in F .
$$

(i) By the property of $\epsilon_{j}$-pseudoconvexity of $f_{j}$, we get

$$
f(z) \leq f(x)+\sqrt{\alpha}\|x-\bar{z}\|
$$

for all $x \in F$. If $\bar{z} \in F_{j}(\bar{z})$ then $\bar{z}$ is an $\epsilon_{j}$-quasisolution of $\left(P_{j}(\bar{z})\right)$.

(ii) If $\bar{z} \in F_{j}^{\epsilon_{j}}(\bar{z})$ then the conclusion (ii) follows.

4.2. Relationships between $\left(D_{j}\right.$ and $\mathrm{MD}$

Proposition 4.7. Suppose that the function $L_{j}(\cdot, \lambda)$ is $\epsilon_{j}$-pseudoconvex. Let $\bar{\tau} \in \mathbb{R}_{+}^{m}$ be such that $\bar{\tau}_{j}=1$ and $\bar{\tau}_{k}=0$ for $k \neq j$.

(i) If $(\bar{z}, \bar{\lambda})$ is an $\epsilon_{j}$-solution of $\left(D_{j}\right)$ then $(\bar{z}, \bar{\lambda}, \bar{\tau})$ is a weakly $\epsilon$-Pareto solution of (MD).

(ii) If $(\bar{z}, \bar{\lambda})$ is an $\epsilon_{j}$-quasisolution of $\left(\overline{D_{j}}\right.$ then $(\bar{z}, \bar{\lambda}, \bar{\tau})$ is a weakly $\epsilon$-Pareto quasisolution of $\mathrm{MD}$.

(iii) If $(\bar{z}, \bar{\lambda})$ is a regular $\epsilon_{j}$-solution of $D_{j}$ then $(\bar{z}, \bar{\lambda}, \bar{\tau})$ is a regular weakly $\epsilon$-Pareto quasisolution of $\mathrm{MD}$.

Proof. (i) Let $(\bar{z}, \bar{\lambda})$ be an $\epsilon_{j}$-solution of $\left.D_{j}\right)$. Hence,

$$
0 \in \partial^{c} f_{j}(\bar{z})+\sum_{t \in T} \bar{\lambda}_{t} \partial^{c} g_{t}(\bar{z})+N_{C}(\bar{z})+\sqrt{\epsilon_{j}} B^{*}
$$

and

$$
L_{j}(z, \bar{\lambda}) \geq L_{j}(y, \lambda)-\epsilon_{j}
$$


for all feasible point $(y, \lambda)$ of $\left(D_{j}\right)$. Let $\bar{\tau} \in \mathbb{R}_{+}^{m}$ be such that $\bar{\tau}_{k}=0$ if $k \neq j$ and $\bar{\tau}_{k}=1$ for $k=j$. Then $(\bar{z}, \bar{\lambda}, \bar{\tau})$ is a feasible point of $(\mathrm{MD})$. We claim that $(\bar{z}, \bar{\lambda}, \bar{\tau})$ is a weakly $\epsilon$-Pareto solution for (MD). On the contrary, if

$$
L_{k}(y, \lambda)-\epsilon_{k}>L_{k}(\bar{z}, \bar{\lambda}), \quad \forall k \in M
$$

holds for some feasible point $(y, \lambda, \tau)$ of $(\mathrm{MD})$, then, this inequality contradicts (4.7).

(ii) Let $(\bar{z}, \bar{\lambda})$ be a quasi $\epsilon_{j}$-solution of $\left(D_{j}\right)$. Hence,

$$
0 \in \partial^{c} f_{j}(\bar{z})+\sum_{t \in T} \bar{\lambda}_{t} \partial^{c} g_{t}(\bar{z})+N_{C}(\bar{z})+\sqrt{\epsilon_{j}} B^{*}
$$

and

$$
L_{j}(\bar{z}, \bar{\lambda}) \geq L_{j}(y, \lambda)-\sqrt{\epsilon_{j}}\|\bar{z}-y\|-\sqrt{\epsilon_{j}}\|\bar{\lambda}-\lambda\|_{1}, \quad \forall(y, \lambda) \in F_{j}^{D} .
$$

Let $\bar{\tau} \in \mathbb{R}_{+}^{m}$ be such that $\bar{\tau}_{k}=0$ if $k \neq j$ and $\bar{\tau}_{k}=1$ for $k=j$. Then $(\bar{z}, \bar{\lambda}, \bar{\tau})$ is a feasible point of (MD). If $(\bar{z}, \bar{\lambda}, \bar{\tau})$ is not a weakly $\epsilon$-Pareto quasisolution of (MD) then there exists a feasible point $(x, \lambda, \tau)$ of $(\mathrm{MD})$ such that, for any $i \in M$,

$$
L_{i}(x, \lambda)-\sqrt{\epsilon_{i}}\|x-\bar{z}\|-\sqrt{\epsilon_{i}}\|\bar{\lambda}-\lambda\|_{1}>L_{i}(\bar{z}, \bar{\lambda}) .
$$

Using an argument similar to the one above, we can easily derive a contradiction.

(iii) The conclusion can be derived by combining (i) and (ii).

Proposition 4.8. Suppose that the functions $L_{j}(\cdot, \lambda)$ are $\epsilon_{j}$-pseudoconvex for $j \in M$. Let $\bar{\tau} \in \mathbb{R}_{++}^{m}$ be such that $\sum_{j \in M} \bar{\tau}_{j}=1$.

(i) If $(\bar{z}, \bar{\lambda})$ is an $\epsilon_{j}$-solution of $\left(D_{j}\right.$ ) for each $j \in M$ then $(\bar{z}, \bar{\lambda}, \bar{\tau})$ is an $\epsilon$-Pareto solution of $\mathrm{MD}$.

(ii) If $(\bar{z}, \bar{\lambda})$ is an $\epsilon_{j}$-quasisolution of $D_{j}$ for each $j \in M$ then $(\bar{z}, \bar{\lambda}, \bar{\tau})$ is an $\epsilon$-Pareto quasisolution of $\mathrm{MD}$.

(iii) If $(\bar{z}, \bar{\lambda})$ is a regular $\epsilon_{j}$-solution of $\left(D_{j}\right.$ for each $j \in M$ then $(\bar{z}, \bar{\lambda}, \bar{\tau})$ is a regular $\epsilon$-Pareto solution of $\mathrm{MD}$.

Proof. (i) Suppose that $(\bar{z}, \bar{\lambda})$ is an $\epsilon_{j}$-solution of $\left[D_{j}\right]$ for each $j \in M$. Then,

$$
0 \in \partial^{c} f_{j}(\bar{z})+\sum_{t \in T} \bar{\lambda}_{t} \partial^{c} g_{t}(\bar{z})+N_{C}(\bar{z})+\sqrt{\epsilon_{j}} B^{*}, \quad j \in M
$$

and

$$
L_{j}(\bar{z}, \bar{\lambda}) \geq L_{j}(y, \lambda)-\epsilon_{j}, \quad \forall(y, \lambda) \in F_{j}^{D} .
$$


Hence, there exist $u_{j} \in \partial^{c} f_{j}(\bar{z}), v_{t}^{j} \in \partial^{c} g_{t}(\bar{z}), t \in T, w^{j} \in N_{C}(\bar{z}), b^{j} \in B^{*}$ such that

$$
0=u^{j}+\sum_{t \in T} \bar{\lambda}_{t} v_{t}^{j}+w^{j}+\sqrt{\epsilon_{j}} b^{j}, \quad j \in M
$$

For $\bar{\tau} \in \mathbb{R}_{++}^{m}$ and $\sum_{j \in M} \bar{\tau}_{j}=1$, from each equality above, we get

$$
0=\bar{\tau}_{j} u^{j}+\sum_{t \in T} \bar{\lambda}_{t} \bar{\tau}_{j} v_{t}^{j}+\bar{\tau}_{j} w^{j}+\bar{\tau}_{j} \sqrt{\epsilon_{j}} b^{j}, \quad j \in M .
$$

Adding the equalities above side by side, we obtain

$$
0=\sum_{j \in M} \bar{\tau}_{j} u^{j}+\sum_{j \in M} \sum_{t \in T} \bar{\lambda}_{t} \bar{\tau}_{j} v_{t}^{j}+\sum_{j \in M} \bar{\tau}_{j} w^{j}+\sum_{j \in M} \bar{\tau}_{j} \sqrt{\epsilon_{j}} b^{j} .
$$

Since $N_{C}(\bar{z})$ and $\partial^{c} g_{t}(\bar{z})$ are convex, there exists $w \in N_{C}(\bar{z})$ such that $w=\sum_{j \in M} \bar{\tau}_{j} w^{j}$ and there exists $v_{t} \in \partial^{c} g_{t}(\bar{z})$ such that $v_{t}=\sum_{j \in M} \bar{\tau}_{j} v_{t}^{j}$. The equality (4.9) becomes

$$
0=\sum_{j \in M} \bar{\tau}_{j} u^{j}+\sum_{t \in T} \bar{\lambda}_{t} v_{t}+w+\sum_{j \in M} \bar{\tau}_{j} \sqrt{\epsilon_{j}} b^{j},
$$

i.e.,

$$
0 \in \sum_{j \in M} \bar{\tau}_{j} \partial^{c} f_{j}(\bar{z})+\sum_{t \in T} \bar{\lambda}_{t} \partial^{c} g_{t}(\bar{z})+N_{C}(\bar{z})+\sum_{j \in M} \bar{\tau}_{j} \sqrt{\epsilon_{j}} B^{*}
$$

Thus, $(\bar{z}, \bar{\lambda}, \bar{\tau})$ is a feasible solution of $(\mathrm{MD})$. If $(\bar{z}, \bar{\lambda}, \bar{\tau})$ is not an $\epsilon$-Pareto solution of (MD) then there exists a feasible point $(y, \lambda, \tau)$ of (MD) such that

$$
\mathcal{L}(y, \lambda)-\epsilon \geq \mathcal{L}(\bar{z}, \bar{\lambda})
$$

and $L_{k}(y, \lambda)-\epsilon_{k}>L_{k}(\bar{z}, \bar{\lambda})$ with some $k \in M$, a contradiction to (4.8). This guarantees that $(\bar{z}, \bar{\lambda}, \bar{\tau})$ is an $\epsilon$-Pareto solution of $(\mathrm{MD})$.

(ii) Suppose that $(\bar{z}, \bar{\lambda})$ is an $\epsilon_{j}$-quasisolution of $\left(D_{j}\right)$ for each $j \in M$. Then,

$$
0 \in \partial^{c} f_{j}(\bar{z})+\sum_{t \in T} \bar{\lambda}_{t} \partial^{c} g_{t}(\bar{z})+N_{C}(\bar{z})+\sqrt{\epsilon_{j}} B^{*},
$$

and for all feasible point $(y, \lambda)$ of $\left[D_{j}\right]$ we get

$$
L_{j}(\bar{z}, \bar{\lambda}) \geq L_{j}(y, \lambda)-\sqrt{\epsilon_{j}}\|\bar{z}-y\|-\sqrt{\epsilon_{j}}\|\bar{\lambda}-\lambda\|_{1}, \quad \forall(y, \lambda) \in F_{j}^{D}, \forall i \in M .
$$

Using an argument similar to the one as above, we get $(\bar{z}, \bar{\lambda}, \bar{\tau})$ is a feasible solution of $(\mathrm{MD})$. If $(\bar{z}, \bar{\lambda}, \bar{\tau})$ is not an $\epsilon$-Pareto quasisolution of (MD) then there exists a feasible point $(y, \lambda, \tau)$ of $(\mathrm{MD})$ such that

$$
L_{i}(y, \lambda)-\sqrt{\epsilon_{i}}\|x-\bar{z}\|-\sqrt{\epsilon_{i}}\|\bar{\lambda}-\lambda\|_{1} \geq L_{i}(\bar{z}, \bar{\lambda}), \quad 1 \leq i \leq m,
$$

with at least one strict inequality. Suppose that the strict inequality holds with $i=k$. We get a contradiction to 4.10.

(iii) The last conclusion can be obtained by combining (i) and (ii). 
Theorem 4.9. Let $(\bar{z}, \bar{\lambda}, \bar{\tau})$ be a feasible solution of $(\mathrm{MD})$, where $\bar{\tau} \in \mathbb{R}_{++}^{m}$ and $\sum_{j \in M} \bar{\tau}_{j}=$ 1.

(i) If $(\bar{z}, \bar{\lambda}, \bar{\tau})$ is an $\epsilon$-Pareto solution of $\mathrm{MD}$ then $(\bar{z}, \bar{\lambda})$ is an $\epsilon$-solution of $\left(D_{j}\right)$ for each $j \in M$.

(ii) If $(\bar{z}, \bar{\lambda}, \bar{\tau})$ is an $\epsilon$-Pareto quasisolution of (MD), then $(\bar{z}, \bar{\lambda})$ is an $\epsilon_{j}$-quasisolution of $D_{j}$ for each $j \in M$.

(iii) If $(\bar{z}, \bar{\lambda}, \bar{\tau})$ is a regular $\epsilon$-Pareto solution of $\left(\mathrm{MD}\right.$ then $(\bar{z}, \bar{\lambda})$ is regular $\epsilon_{j}$-solution of $\left(D_{j}\right.$ for each $j \in M$.

Proof. First, we will prove that if $(\bar{z}, \bar{\lambda}, \bar{\tau})$ is a feasible solution of (MD) then $(\bar{z}, \bar{\lambda})$ is a feasible solution of $\left(D_{j}\right)$ for every $j \in M$.

If $(\bar{z}, \bar{\lambda}, \bar{\tau})$ is a feasible solution for $\mathrm{MD}$ with $0<\bar{\tau} \in \mathbb{R}^{m}$ and $\sum_{j \in M} \bar{\tau}_{j}=1$, we get

$$
0 \in \sum_{j \in M} \bar{\tau}_{j} \partial^{c} f_{j}(\bar{z})+\sum_{t \in T} \bar{\lambda}_{t} \partial^{c} g_{t}(\bar{z})+N_{C}(\bar{z})+\sum_{j \in M} \bar{\tau}_{j} \sqrt{\epsilon_{j}} B^{*} .
$$

Let $K$ be the set of index $k \in M$ such that $(\bar{z}, \bar{\lambda})$ is not feasible solution for $\left(D_{k}\right)$ and assume that $K$ is not empty. Then,

$$
0 \notin \partial^{c} f_{k}(\bar{z})+\sum_{t \in T} \bar{\lambda}_{t} \partial^{c} g_{t}(\bar{z})+N_{C}(\bar{z})+\sqrt{\epsilon_{k}} B^{*}, \quad k \in K
$$

and

$$
0 \in \partial^{c} f_{i}(\bar{z})+\sum_{t \in T} \bar{\lambda}_{t} \partial^{c} g_{t}(\bar{z})+N_{C}(\bar{z})+\sqrt{\epsilon_{i}} B^{*}, \quad \forall i \in M \backslash K
$$

Hence,

$$
0 \notin \bar{\tau}_{k} \partial^{c} f_{k}(\bar{z})+\bar{\tau}_{k} \sum_{t \in T} \bar{\lambda}_{t} \partial^{c} g_{t}(\bar{z})+\bar{\tau}_{k} N_{C}(\bar{z})+\bar{\tau}_{k} \sqrt{\epsilon_{k}} B^{*}, \quad \forall k \in K,
$$

and we get

$$
0 \in \bar{\tau}_{i} \partial^{c} f_{i}(\bar{z})+\bar{\tau}_{i} \sum_{t \in T} \bar{\lambda}_{t} \partial^{c} g_{t}(\bar{z})+\bar{\tau}_{i} N_{C}(\bar{z})+\bar{\tau}_{i} \sqrt{\epsilon_{i}} B^{*}, \quad \forall i \in M \backslash K .
$$

From 4.12 and 4.13, by adding side by side, we obtain

$$
0 \notin \sum_{j \in M} \bar{\tau}_{j} \partial^{c} f_{j}(\bar{z})+\sum_{t \in T} \bar{\lambda}_{t} \partial^{c} g_{t}(\bar{z})+N_{C}(\bar{z})+\sum_{j \in M} \bar{\tau}_{j} \sqrt{\epsilon_{j}} B^{*},
$$

a contradiction to 4.11).

(i) If $(\bar{z}, \bar{\lambda})$ is not an $\epsilon_{j}$-solution for $\left(D_{j}\right)$ then there exists $(y, \lambda)$ such that

$$
L_{j}(\bar{z}, \bar{\lambda})<L_{j}(y, \lambda)-\epsilon_{j},
$$


a contradiction to (i) of Definition 4.1 .

(ii) If $(\bar{z}, \bar{\lambda})$ is not an $\epsilon_{j}$-quasisolution for $D_{j}$ then there exists $(y, \lambda)$ such that

$$
L_{j}(\bar{z}, \bar{\lambda})<L_{j}(y, \lambda)-\sqrt{\epsilon_{j}}\|\bar{z}-y\|-\sqrt{\epsilon_{j}}\|\bar{\lambda}-\lambda\|_{1},
$$

a contradiction to (ii) of Definition 4.1

(iii) The conclusion follows by (i) and (ii).

Theorem 4.10. Suppose that $L_{j}(\cdot, \lambda)$ is $\epsilon_{j}$-pseudoconvex and $f_{j}, g_{t}$ are regular on $C$ for $t \in T$. For $j \in M$, suppose that there exists $(\bar{z}, \bar{\lambda}) \in C \times \mathbb{R}_{+}^{(T)}$ satisfying the strict generalized $j-K K T$ condition up to $\epsilon_{j}$ given by $(3.3)$. Then, $(\bar{z}, \bar{\lambda}, \bar{\tau})$ is a weakly $\epsilon$-Pareto quasisolution of $\mathrm{MD}$ where $\bar{\tau}_{j}=1$ and $\bar{\tau}_{k}=0$ for $k \neq j$.

Proof. Since $(\bar{z}, \bar{\lambda})$ satisfies the condition $(3.3),(\bar{z}, \bar{\lambda})$ is a feasible point of $\left(D_{j}\right)$. Moreover, it is easy to check that $(\bar{z}, \bar{\lambda}, \bar{\tau})$ is a feasible solution of (MD) where $\bar{\tau}_{j}=1$ and $\bar{\tau}_{k}=0$ for $k \neq j$. To prove that $(\bar{z}, \bar{\lambda}, \bar{\tau})$ is a weakly $\epsilon$-Pareto solution of (MD), we will prove that $(\bar{z}, \bar{\lambda})$ is an $\epsilon_{j}$-solution for $\left(D_{j}\right)$. Indeed, since $(\bar{z}, \bar{\lambda}) \in C \times \mathbb{R}_{+}^{(T)}$ satisfying the strict generalized $j$-KKT condition up to $\epsilon_{j}$, using the argument similarly to the one in the proof of Theorem 4.4, we can verify that $(\bar{z}, \bar{\lambda})$ is an $\epsilon_{j}$-quasisolution of $\left(D_{j}\right)$. The conclusion follows by Proposition 4.7 .

Theorem 4.11. Suppose that there exists $j \in M$ such that the function $L_{j}(\cdot, \lambda)$ is $\epsilon_{j}$ pseudoconvex and the functions $g_{t}$ are regular for $t \in T$. Then there exists a quasi weakly $\epsilon$-Pareto solution of (MD).

Proof. Let us consider the problem $\left[D_{j}\right.$ c corresponding to $\mathrm{MD}$ and the problem $P_{j}(y)$ corresponding to $\left(D_{j}\right)$. By Lemma 3.5, there exists an almost regular $\epsilon_{j}$-solution $\bar{z}$ for $\left(P_{j}(\bar{z})\right)$ and $\bar{\lambda} \in \mathbb{R}_{+}^{(\bar{T})}$ such that the pair $(\bar{z}, \bar{\lambda})$ is a strict generalized $j$-KKT pair up to $\epsilon_{j}$. By Corollary $4.5,(\bar{z}, \bar{\lambda})$ is an $\epsilon_{j}$-quasisolution of $\left.D_{j}\right)$. The desired result derives by applying Proposition 4.7 .

\subsection{Duality relationships between $(\mathrm{MP})$ and $\mathrm{MD}$}

Theorem 4.12. Suppose that $f_{j}$ and $L_{j}(\cdot, \lambda)$ are $\epsilon_{j}$-pseudoconvex for each $j \in M$, and $g_{t}$ are quasiconvex for all $t \in T$. Let $x$ and $(y, \lambda, \tau)$ be feasible solutions of $(\mathrm{MP}$ and $(\mathrm{MD}$, respectively. Then, the following cannot hold:

$$
\left(f_{1}(x), \ldots, f_{m}(x)\right)<\left(L_{1}(y)-\sqrt{\epsilon_{1}}\|x-y\|, \ldots, L_{m}(y)-\sqrt{\epsilon_{m}}\|x-y\|\right) .
$$

Proof. Let $(y, \lambda, \tau)$ be a feasible solution of $\left(\mathrm{MD}\right.$. There exist $v_{t} \in \partial^{c} g_{t}(y), t \in T$, $w \in N_{C}(y), u_{j} \in \partial^{c} f_{j}(y), b \in B^{*}$ such that

$$
\sum_{t \in T} \lambda_{t} v_{t}+w+\sum_{j \in M} \tau_{j}\left[u_{j}+\sqrt{\epsilon_{j}} b\right]=0 .
$$


Hence,

$$
\sum_{t \in T} \lambda_{t} v_{t}(x-y)+\sum_{j \in M} \tau_{j}\left[u_{j}(x-y)+\sqrt{\epsilon_{j}}\|x-y\|\right] \geq 0, \quad \forall x \in C .
$$

Suppose contrary to 4.14 that

$$
f_{i}(x)<L_{i}(y)-\sqrt{\epsilon_{i}}\|x-y\|, \quad i \in M
$$

i.e.,

$$
f_{i}(x)+\sqrt{\epsilon_{i}}\|x-y\|<f_{i}(y)+\sum_{t \in T} \lambda_{t} g_{t}(y), \quad i \in M .
$$

For $x \in F$, we get $\lambda_{t} g_{t}(x) \leq 0$ for all $t \in T$. Then

$$
f_{i}(x)+\sum_{t \in T} \lambda_{t} g_{t}(x)+\sqrt{\epsilon_{i}}\|x-y\|<f_{i}(y)+\sum_{t \in T} \lambda_{t} g_{t}(y), \quad i \in M .
$$

Since $L_{i}(\cdot, \lambda)$ is $\epsilon_{i}$-pseudoconvex, the inequality above implies that

$$
L_{i}^{\circ}(y ; x-y)+\sqrt{\epsilon_{i}}\|x-y\|<0, \quad i \in M .
$$

Hence, $u_{i}(x-y)+\sum_{t \in T} \lambda_{t} v_{t}(x-y)+\sqrt{\epsilon_{i}}\|x-y\|<0, i \in M$. Thus,

$$
\sum_{t \in T} \lambda_{t} v_{t}(x-y)+\sum_{i \in M} \tau_{i}\left[u_{i}(x-y)+\sqrt{\epsilon_{i}}\|x-y\|\right]<0 .
$$

This and 4.15) imply a contradiction.

We now propose some theorems to describe the relationships between (MP) and (MD) via a pair of primal-dual scalar problems.

Theorem 4.13. Suppose that $L_{j}(\cdot, \lambda)$ is $\epsilon_{j}$-pseudoconvex and $f_{j}, g_{t}$ are regular on $C$ for $t \in T$. Let $\epsilon \in \mathbb{R}_{++}^{m}$. Suppose further that $\bar{z}$ is an almost $\epsilon$-Pareto quasisolution of (MP) and there exist $j \in M, \bar{\lambda} \in \mathbb{R}_{+}^{(T)}$, such that $(\bar{z}, \bar{\lambda})$ is a strict generalized $j-K K T$ pair up to $\epsilon_{j}$ then $(\bar{z}, \bar{\lambda}, \bar{\tau})$ is a weakly $\epsilon$-Pareto quasisolution of (MD) where $\tau_{j}=1$ and $\tau_{k}=0$ with $k \neq j$.

Proof. Let $\epsilon \in \mathbb{R}_{++}^{m}$. Suppose that $\bar{z}$ is an almost $\epsilon$-Pareto quasisolution of (MP). Hence, $\bar{z}$ is an almost $\epsilon_{j}$-quasisolution for $\left(P_{j}(\bar{z})\right)$ for each $j \in M$. Since, there exist $j \in M$ and $\bar{\lambda}$ such that $(\bar{z}, \bar{\lambda})$ is a strict generalized $j$-KKT pair up to $\epsilon_{j}$, by using an argument similar to the one in the proof of Theorem 4.4 , we get $(\bar{z}, \bar{\lambda})$ is an $\epsilon_{j}$-quasisolution for $\left(D_{j}\right]$. The desired result follows by Proposition 4.7 .

Theorem 4.14. Let $\epsilon>0, \epsilon \in \mathbb{R}^{m}$ and let $(\bar{z}, \bar{\lambda}, \bar{\tau})$ be a quasi $\epsilon$-Pareto solution for (MD, where $\bar{\tau} \in \mathbb{R}_{++}^{m}$ and $\sum_{j \in M} \bar{\tau}_{j}=1$. 
(i) If $\bar{z} \in F_{j}(\bar{z})$ then $\bar{z}$ is a weakly $\epsilon$-Pareto quasisolution of $(\mathrm{MP}$.

(ii) For $\epsilon_{j}=\min \left\{\epsilon_{k}, k \in M\right\}$, if $\bar{z} \in F_{j}^{\epsilon_{j}}(\bar{z})$ then $\bar{z}$ is an almost weakly $\epsilon$-Pareto quasisolution of $\mathrm{MP}$.

Proof. Let $\epsilon>0, \epsilon \in \mathbb{R}^{m}$. Suppose that $(\bar{z}, \bar{\lambda}, \bar{\tau})$ is an $\epsilon$-Pareto quasisolution for (MD), where $0<\bar{\tau} \in \mathbb{R}^{m}$ and $\sum_{j \in M} \bar{\tau}_{j}=1$. By Theorem 4.9. $(\bar{z}, \bar{\lambda})$ is an $\epsilon_{j}$-quasisolution of D $D_{j}$ for each $j \in M$.

If $\bar{z} \in F_{j}(\bar{z})\left(\bar{z} \in F_{j}^{\epsilon_{j}}(\bar{z})\right)$, by Theorem 4.6 , it is an (almost) $\epsilon_{j}$-quasisolution of $\left(P_{j}(\bar{z})\right)$. For $\epsilon_{j}=\min \left\{\epsilon_{k} \mid k \in M\right\}$, the conclusions can be derived by Theorem 3.7 .

4.4. A modification of Chankong-Haimes method for a dual problem of $\mathrm{MP}$

Note that, if we deal with exact solution of $(\mathrm{MP})$ then a dual problem of $(\mathrm{MP})$ in Wolfe type is formulated by

$\left(\mathrm{MD}^{*}\right)$

$$
\begin{array}{r}
\operatorname{Max} \mathcal{L}(y, \lambda), \quad 0 \in \sum_{j \in M} \tau_{j} \partial^{c} f_{j}(y)+\sum_{t \in T} \lambda_{t} \partial^{c} g_{t}(y)+N_{C}(y), \\
\tau_{j} \geq 0, \quad \sum_{j \in M} \tau_{j}=1, \quad y \in C, \quad \lambda \in \mathbb{R}_{+}^{(T)} .
\end{array}
$$

Hence, the problem $D_{j}, j=1,2$, becomes

$$
\begin{gathered}
\operatorname{Max} L_{j}(y, \lambda)=f_{j}(y)+\sum_{t \in T} \lambda_{t} g_{t}(y) \\
0 \in \partial^{c} f_{j}(y)+\sum_{t \in T} \lambda_{t} \partial^{c} g_{t}(y)+N_{C}(y), \quad(y, \lambda) \in C \times \mathbb{R}_{+}^{(T)} .
\end{gathered}
$$

The problem $\left(D_{j}^{*}\right)$ is not compatible with $\left(P_{j}\right)$ under the dual scheme of Wolfe type. But, there exist connections between $\left(D_{j}^{*}\right)$ and $\left(\mathrm{MD}^{*}\right)$, directly. Approximate solutions of (MD* can be obtained via $\left(D_{j}^{*}\right)$ in the spirit of Chankong-Haimes method. We propose some more propositions with the proofs omitted because they are similar to the proofs of Propositions 4.7(i), 4.8(i) and Theorem 4.9(i), respectively.

Proposition 4.15. Suppose that the function $L_{j}(\cdot, \lambda)$ is $\epsilon_{j}$-pseudoconvex. Let $\bar{\tau} \in \mathbb{R}_{+}^{m}$ be such that $\bar{\tau}_{j}=1$ and $\bar{\tau}_{k}=0$ for $k \neq j$. If $(\bar{z}, \bar{\lambda})$ is an $\epsilon_{j}$-solution of $D_{j}^{*}$ then $(\bar{z}, \bar{\lambda}, \bar{\tau})$ is a weakly $\epsilon$-Pareto solution of (MD*).

Proposition 4.16. Suppose that the functions $L_{j}(\cdot, \lambda)$ are $\epsilon_{j}$-pseudoconvex for $j \in M$. Let $\bar{\tau} \in \mathbb{R}_{++}^{m}$ be such that $\sum_{j \in M} \bar{\tau}_{j}=1$. If $(\bar{z}, \bar{\lambda})$ is an $\epsilon_{j}$-solution of $\left(D_{j}^{*}\right)$ for each $j \in M$ then $(\bar{z}, \bar{\lambda}, \bar{\tau})$ is an $\epsilon$-Pareto solution of $\mathrm{MD}^{*}$.

Proposition 4.17. Let $(\bar{z}, \bar{\lambda}, \bar{\tau})$ be a feasible solution of $\mathrm{MD}^{*}$, where $\bar{\tau} \in \mathbb{R}_{++}^{m}$ and $\sum_{j \in M} \bar{\tau}_{j}=1$. If $(\bar{z}, \bar{\lambda}, \bar{\tau})$ is an $\epsilon$-Pareto solution of $\left(\mathrm{MD}^{*}\right.$ then $(\bar{z}, \bar{\lambda})$ is an $\epsilon$-solution of D $D_{j}^{*}$ for each $j \in M$. 
Example 4.18. The following example illustrates Proposition 4.15.

$$
\text { Min } f(x)=\left(x, x^{2}-x\right) \text { subject to } g(x)=x^{2}-x \leq 0 \text {. }
$$

The dual problem of $\left(\mathrm{MP}_{1}\right)$ in Wolfe type, following (MD), is

$$
\begin{aligned}
& \operatorname{Max} L(y, \lambda)=\left(y+\lambda\left(y^{2}-y\right), y^{2}-y+\lambda\left(y^{2}-y\right)\right) \\
& \text { subject to } 0=\tau_{1}+\tau_{2}(2 y-1)+\lambda(2 y-1) \\
& \tau_{1}, \tau_{2} \geq 0, \quad \tau_{1}+\tau_{2}=1, \quad y \in \mathbb{R}, \quad \lambda \in \mathbb{R}_{+} .
\end{aligned}
$$

Given $\epsilon=\left(\epsilon_{1}, \epsilon_{2}\right)=(1 / 4,1 / 4)$, it is easy to check that the following functions are $\epsilon_{j^{-}}$ pseudoconvex $(j=1,2)$, respectively:

$$
L_{1}(y, \lambda)=y+\lambda\left(y^{2}-y\right), \quad L_{2}(y, \lambda)=y^{2}-y+\lambda\left(y^{2}-y\right) .
$$

The problems $\left(D_{1}^{*}\right)$ and $\left(D_{2}^{*}\right)$ formulated due to $\left(D_{j}^{*}\right]$ are as follows:

$\left(D_{1}^{*}\right)$ Maximize $L_{1}(y, \lambda)=y+\lambda\left(y^{2}-y\right)$ subject to $0=1+\lambda(2 y-1), y \in \mathbb{R}, \lambda \in \mathbb{R}_{+}$,

$$
\text { Maximize } L_{2}(y, \lambda)=y^{2}-y+\lambda\left(y^{2}-y\right)
$$

$$
\text { subject to } 0=2 y-1+\lambda(2 y-1), y \in \mathbb{R}, \lambda \in \mathbb{R}_{+} \text {. }
$$

For $\left(D_{1}^{*}\right)$, it is easy to check that $(y, \lambda)=(0,1)$ its an exact solution and the optimal value of the problem is 0 . With a simple computation, we can see that each pair $(z, \bar{\lambda})$, where $z \in[-(1+\sqrt{5}) / 4,(-1+\sqrt{5}) / 4]$ and $\bar{\lambda}=1 /(1-2 z)$, is a $1 / 4$-solution of $\left(D_{1}^{*}\right)$. In this case, the quadruple $\left(z, \bar{\lambda}, \tau_{1}, \tau_{2}\right)$ where $z \in[-(1+\sqrt{5}) / 4,(-1+\sqrt{5}) / 4], \bar{\lambda}=1 /(1-2 z)$, $\tau_{1}=1, \tau_{2}=0$ is a weakly $\epsilon$-Pareto solution of $\left(\mathrm{MD}_{1}^{*}\right)$.

Also, for $\left.D_{2}^{*}\right)$, we can check that $(y, \lambda)=(1 / 2,0)$ is an exact solution and the optimal value is $-1 / 4$. Each pair $(z, \bar{\lambda})$, where $z=1 / 2$ and $\bar{\lambda} \in[0,1]$, is a $1 / 4$-solution of $D_{2}^{*}$. Hence, the quadruple $\left(z, \bar{\lambda}, \tau_{1}, \tau_{2}\right)$ where $z=1 / 2, \bar{\lambda} \in[0,1], \tau_{1}=0, \tau_{2}=1$ is a weakly $\epsilon$-Pareto solution of $\left(\mathrm{MD}_{1}^{*}\right)$.

\section{Conclusions}

We proposed an approach to investigate $\epsilon$-duality theorems for a class of semi-infinite multiobjective optimization problems via a pair of primal-dual scalar problems. This approach is based on a development of Chankong-Haimes method [5] and it differs from the weighted-sum method for solving multiobjective optimization problems introduced in 38. The relations among four problems, the primal-dual multiobjective problems and the primal-dual scalar problems, are established. Then, we give $\epsilon$-duality theorems due to the symmetric diagram proposed in Section 1. At the end of the paper, we show that 
approximate Pareto solution of the dual problem of (MP) can be obtained directly by the spirit of Chankong-Haimes method applied for the dual problem of (MP). As an application, we state that this scheme can be applied for fractional semi-infinite multiobjective optimization problems by the use of a well known Dinkelbach transformation (to change a fractional optimization problem to a nonfractional optimization problem). We leave this for our further research.

\section{Acknowledgments}

The authors would like to express their sincere thanks to anonymous referees for their valuable suggestions and comments. Besides, the authors would like to thank Dr. Liguo Jiao for some remarkable discussions and comments in the revised version. The first author was supported by the National Research Foundation of Korea Grant funded by the Korean Government (NRF-2016R1A2B4011589). The second author was supported by Vietnam National Foundation for Science and Technology Development (NAFOSTED) under grant number 101.01-2017.08.

\section{References}

[1] M. Beldiman, E. Panaitescu and L. Dogaru, Approximate quasi efficient solutions in multiobjective optimization, Bull. Math. Soc. Sci. Math. Roumanie (N.S.) 51 (2008), no. 2, 109-121.

[2] R. I. Bot, S.-M. Grad and G. Wanka, Duality in Vector Optimization, Vector Optimization, Springer-Verlag, Berlin, 2009.

[3] E. Carrizosa, E. Conde, M. Muñoz-Márquez and J. Puerto, Planar point-objective location problems with nonconvex constraints: a geometrical construction, J. Global Optim. 6 (1995), no. 1, 77-86.

[4] S. Chandra, J. Dutta and C. S. Lalitha, Regularity conditions and optimality in vector optimization, Numer. Funct. Anal. Optim. 25 (2004), no. 5-6, 479-501.

[5] V. Chankong and Y. Y. Haimes, Multiobjective Decision Making: Theory and Methodology, North-Holland Series in System Science and Engineering 8, North-Holland, New York, 1983.

[6] T. D. Chuong, Nondifferentiable fractional semi-infinite multiobjective optimization problems, Oper. Res. Lett. 44 (2016), no. 2, 260-266. 
[7] T. D. Chuong and D. S. Kim, Nonsmooth semi-infinite multiobjective optimization problems, J. Optim. Theory Appl. 160 (2014), no. 3, 748-762.

[8] _ Approximate solutions of multiobjective optimization problems, Positivity 20 (2016), no. 1, 187-207.

[9] F. H. Clarke, Optimization and Nonsmooth Analysis, Canadian Mathematical Society Series of Monographs and Advanced Texts, A Wiley-Interscience, John Wiley \& Sons, New York, 1983.

[10] F. H. Clarke, Y. S. Ledyaev, R. J. Stern and P. R. Wolenski, Nonsmooth Analysis and Control Theory, Graduate Texts in Mathematics 178, Spinger-Verlag, Berlin, 1998.

[11] J. Dutta, Necessary optimality conditions and saddle points for approximate optimization in Banach spaces, TOP 13 (2005), no. 1, 127-143.

[12] M. Ehrgott, Multicriteria Optimization, Second edition, Springer-Verlag, Berlin, 2005.

[13] G. Eichfelder, Adaptive Scalarization Methods in Multiobjective Optimization, Vector Optimization, Springer-Verlag, Berlin, 2008.

[14] A. Engau and M. M. Wiecek, Exact generation of epsilon-efficient solutions in multiple objective programming, OR Spectrum 29 (2007), no. 2, 335-350.

[15] - Cone characterizations of approximate solutions in real vector optimization, J. Optim. Theory Appl. 134 (2007), no. 3, 499-513.

[16] G. Giorgi, A. Guerraggio and J. Thierfelder, Mathematics of Optimization: Smooth and Nonsmooth Case, Elsevier Science B.V., Amsterdam, 2004.

[17] M. A. Goberna and M. A. López, Linear Semi-infinite Optimization, Wiley Series in Mathematical Methods in Practice 2, John Wiley \& Sons, Chichester, 1998.

[18] M. G. Govil and A. Mehra, $\epsilon$-optimality for multiobjective programming on a Banach space, European J. Oper. Res. 157 (2004), no. 1, 106-112.

[19] P. Gupta, S. Shiraishi and K. Yokoyama, e-optimality without constraint qualification for multiobjective fractional program, J. Nonlinear Convex Anal. 6 (2005), no. 2, $347-357$.

[20] C. Gutiérrez, B. Jiménez and V. Novo, On approximate solutions in vector optimization problems via scalarization, Comput. Optim. Appl. 35 (2006), no. 3, 305-324. 
[21]__ Optimality conditions for metrically consistent approximate solutions in vector optimization, J. Optim. Theory Appl. 133 (2007), no. 1, 49-64.

[22] A. Hamel, An $\epsilon$-Lagrange multiplier rule for a mathematical programming problem on Banach spaces, Optimization 49 (2001), no. 1-2, 137-149.

[23] J.-B. Hiriart-Urruty, New concepts in nondifferentiable programming, Bull. Soc. Math. France, Mém. 60 (1979), 57-85.

[24] J. Jahn, Scalarization in vector optimization, Math. Programming 29 (1984), no. 2, $203-218$.

[25] _ Vector Optimization: Theory, Applications, and Extensions, Second edition, Springer, 2011.

[26] K. Klamroth, R. Lacour and D. Vanderpooten, On the representation of the search region in multi-objective optimization, European J. Oper. Res. 245 (2015), no. 3, $767-778$.

[27] K. Klamroth and J. Tind, Constrained optimization using multiple objective programming, J. Global Optim. 37 (2007), no. 3, 325-355.

[28] K. O. Kortanek, Constructing a perfect duality in infinite programming, Appl. Math. Optim. 3 (1977), no. 4, 357-372.

[29] X. F. Li, Constraint qualifications in nonsmooth multiobjective optimization, J. Optim. Theory Appl. 106 (2000), no. 2, 373-398.

[30] J. C. Liu, $\epsilon$-duality theorem of nondifferentiable nonconvex multiobjective programming, J. Optim. Theory Appl. 69 (1991), no. 1, 153-167.

[31] P. Loridan, Necessary conditions for E-optimality: Optimality and stability in mathematical programming, Math. Programming Stud. 19 (1982), 140-152.

[32] _ $\ldots$-solutions in vector minimization problems, J. Optim. Theory Appl. 43 (1984), no. 2, 265-276.

[33] D. T. Luc, Scalarization of vector optimization problems, J. Optim. Theory Appl. 55 (1987), no. 1, 85-102.

[34] R. T. Marler and J. S. Arora, Survey of multi-objective optimization methods for engineering, Struct. Multidiscip. Optim. 26 (2004), no. 6, 369-395. 
[35] K. Miettinen, M. M. Mäkelä and K. Kaario, Experiments with classification-based scalarizing functions in interactive multiobjective optimization, European J. Oper. Res. 175 (2006), no. 2, 931-947.

[36] G.-R. Piao, L. Jiao and D. S. Kim, Optimality conditions in nonconvex semi-infinite multiobjective optimization problems, J. Nonlinear Convex Anal. 17 (2016), no. 1, $167-175$.

[37] J.-J. Rückmann and A. Shapiro, Augmented Lagrangians in semi-infinite programming, Math. Program. 116 (2009), no. 1-2, 499-512.

[38] T. Q. Son and D. S. Kim, $\varepsilon$-mixed type duality for nonconvex multiobjective programs with an infinite number of constraints, J. Global Optim. 57 (2013), no. 2, 447-465.

[39] _ A new approach to characterize the solution set of a pseudoconvex programming problem, J. Comput. Appl. Math. 261 (2014), 333-340.

[40] T. Q. Son, D. S. Kim and N. N. Tam, Weak stability and strong duality of a class of nonconvex infinite programs via augmented Lagrangian, J. Global Optim. 53 (2012), no. $2,165-184$.

[41] T. Q. Son, J. J. Strodiot and V. H. Nguyen, $\epsilon$-optimality and $\epsilon$-Lagrangian duality for a nonconvex programming problem with an infinite number of constraints, J. Optim. Theory Appl. 141 (2009), no. 2, 389-409.

Do Sang Kim

Department of Applied Mathematics, Pukyong National University, Busan, 48513, Korea E-mail address: dskim@pknu.ac.kr

Ta Quang Son

Faculty of Mathematics and Applications, Saigon University, Hochiminh City, Vietnam E-mail address: taquangson@sgu.edu.vn 